\title{
ARTICLE
}

Molecular Diagnostics

\section{A model combining clinical and genomic factors to predict response to PD-1/PD-L1 blockade in advanced urothelial carcinoma}

Amin H. Nassar ${ }^{1,2}$, Kent W. Mouw ${ }^{3}$, Opeyemi Jegede ${ }^{4}$, Atul B. Shinagare ${ }^{5}$, Jaegil Kim ${ }^{6}$, Chia-Jen Liu ${ }^{1}$, Mark Pomerantz ${ }^{1}$, Lauren C. Harshman ${ }^{1}$, Eliezer M. Van Allen ${ }^{1}$, Xiao X. Wei ${ }^{1}$, Bradley McGregor ${ }^{1}$, Atish D. Choudhury ${ }^{1}$, Mark A. Preston ${ }^{7}$, Fei Dong ${ }^{8}$, Sabina Signoretti ${ }^{8,9}$, Neal I. Lindeman ${ }^{8}$, Joaquim Bellmunt ${ }^{10}$, Toni K. Choueiri (D) ${ }^{1}$, Guru Sonpavde ${ }^{1}$ and David J. Kwiatkowski ${ }^{1,2}$

\begin{abstract}
BACKGROUND: In metastatic urothelial carcinoma (mUC), predictive biomarkers that correlate with response to immune checkpoint inhibitors (ICls) are lacking. Here, we interrogated genomic and clinical features associated with response to ICls in mUC. METHODS: Sixty two mUC patients treated with $\mathrm{ICl}$ who had targeted tumour sequencing were studied. We examined associations between candidate biomarkers and clinical benefit (CB, any objective reduction in tumour size) versus no clinical benefit (NCB, no change or objective increase in tumour size). Both univariable and multivariable analyses for associations were conducted. A comparator cohort of $39 \mathrm{mUC}$ patients treated with taxanes was analysed by using the same methodology.

RESULTS: Nine clinical and seven genomic factors correlated with clinical outcomes in univariable analysis in the ICl cohort. Among the 16 factors, neutrophil-to-lymphocyte ratio ( $N L R) \geq 5(\mathrm{OR}=0.12,95 \% \mathrm{Cl}, 0.01-1.15)$, visceral metastasis $(\mathrm{OR}=0.05,95 \% \mathrm{Cl}$, $0.01-0.43)$ and single-nucleotide variant (SNV) count $<10(\mathrm{OR}=0.04,95 \% \mathrm{Cl}, 0.006-0.27)$ were identified as independent predictors of NCB to ICI in multivariable analysis (c-statistic $=0.90$ ). None of the 16 variables were associated with clinical benefit in the taxane cohort.

CONCLUSIONS: This three-factor model includes genomic (SNV count $>9$ ) and clinical (NLR $<5$, lack of visceral metastasis) variables predictive for benefit to $\mathrm{ICl}$ but not taxane therapy for mUC. External validation of these hypothesis-generating results is warranted to enable use in routine clinical care.
\end{abstract}

British Journal of Cancer (2020) 122:555-563; https://doi.org/10.1038/s41416-019-0686-0

\section{BACKGROUND}

Immune checkpoint inhibitors (ICls) have transformed the therapeutic landscape of a growing list of human cancers, ${ }^{1-3}$ including metastatic urothelial carcinomas (mUC). ${ }^{4-7}$ However, only $20-30 \%$ of mUC patients respond to $\mathrm{ICls}$, and an even smaller proportion achieve durable responses lasting $\geq 2$ years. ${ }^{8}$ Hence, mUC remains an incurable disease for the majority of patients due to inherent or acquired therapeutic resistance. The mechanisms underlying variation in $\mathrm{ICl}$ response among mUC patients are poorly understood, and there is an urgent clinical need to identify biomarkers that are predictive of $\mathrm{ICl}$ benefit and to elucidate the mechanisms of resistance in $\mathrm{ICl}$ nonresponders.

Currently, the only Food and Drug Administration-approved predictive biomarkers of $\mathrm{ICl}$ response are programmed deathligand (PD-L1) expression for specific cancers and microsatellite instability-high (MSI-H)/mismatch repair deficiency (dMMR) for a tumour-agnostic indication. However, although PD-L1 expression has some value for prediction of response to $\mathrm{ICls}$, it is not consistent across different ICls and lines of therapy., ${ }^{5-11}$ Mismatch repair deficiency is associated with clinical benefit in several tumour types, ${ }^{9,12}$ but is rare in mUC. Tumour mutational burden (TMB) and predicted neoantigen load have also been correlated with $\mathrm{ICl}$ response in several tumour types, including non-small-cell lung cancer and melanoma.,13-17 However, the clinical applicability and predictive power of TMB is uncertain. ${ }^{18}$ In $\mathrm{mUC}$, low $T M B$ does not preclude response and high TMB is not sufficient to predict response. $^{19}$

Recently, DNA damage repair (DDR) gene alterations were reported to be associated with response to ICls in patients with mUC in a single-institution series. ${ }^{19}$ Larger prospective cohorts are required to validate this potential biomarker. In addition, peripheral blood markers have emerged as potential biomarkers of $\mathrm{ICl}$ response in multiple cancer types. Low absolute neutrophil count, low neutrophil-to-lymphocyte ratio (NLR) and low absolute monocyte count were associated with improved overall and progression-free survival in melanoma patients receiving

\footnotetext{
${ }^{1}$ Department of Medical Oncology, Dana-Farber Cancer Institute, Boston, MA, USA; ${ }^{2}$ Department of Medicine, Brigham and Women's Hospital, Boston, MA, USA; ${ }^{3}$ Department of Radiation Oncology, Dana-Farber Cancer Institute, Boston, MA, USA; ${ }^{4}$ Department of Biostatistics and Computational Biology, Dana-Farber Cancer Institute, Harvard Medical School, Boston, MA, USA; ${ }^{5}$ Department of Radiology, Brigham and Women's Hospital, Boston, MA, USA; ${ }^{6}$ The Broad Institute of MIT and Harvard, Cambridge, MA, USA; ${ }^{7}$ Division of Urology, Brigham and Women's Hospital, Boston, MA, USA; ${ }^{8}$ Department of Pathology, Brigham and Women's Hospital, Boston, MA, USA; ${ }^{9}$ Department of Oncologic Pathology, Dana-Farber Cancer Institute, Boston, MA, USA and ${ }^{10}$ Department of Medical Oncology, IMIM-Hospital del Mar Medical Research Institute, Barcelona, Spain Correspondence: Guru Sonpavde (GuruP_Sonpavde@dfci.harvard.edu) or David J. Kwiatkowski (dk@rics.bwh.harvard.edu)
} 
ipilimumab (anti-CTLA-4). ${ }^{20}$ In addition, a recently reported sixfactor prognostic model for overall survival in advanced UC patients treated with post-platinum atezolizumab consisted of two clinical factors (ECOG PS $\geq 1$ vs. 0 , presence of liver metastasis) and four blood-based biomarkers (anaemia, thrombocytosis, NLR $\geq 5$ and elevated $\mathrm{LDH})^{21}$ In this study, we sought to develop an integrated model combining genomic, clinical and routine laboratory factors to predict response to anti-PD-1/PD-L1 inhibitors in $\mathrm{mUC}$ regardless of the setting (i.e. first-line or post platinum).

\section{METHODS}

Study design and patient cohort

We identified patients with histologically confirmed diagnosis of mUC treated with an anti-PD-1/PD-L1 agent at Dana-Farber Cancer Institute (DFCl) between June 2013 and December 2017 who also underwent tumour DNA sequencing analysis (see below) (Fig. S1). Mutational findings in these patients were recently reported but were not analysed for response to immune checkpoint therapy. ${ }^{22}$ A radiologist (A.S.), blinded to genomic and clinical data, performed tumour measurements using Response Evaluation Criteria in Solid Tumors version 1.1 (RECIST v1.1). Both computerised axial tomography and positron emission tomography scans were used to assess measurable lesions (total of five lesions and maximum of two per organ) that met RECIST v1.1 criterion. With prior reports suggesting a need for enhanced clinical endpoints to assess clinical benefit in patients treated with $\mathrm{ICls}^{23}$ patients were classified as having clinical benefit (CB) if they had any objective reduction in tumour burden, and having no clinical benefit $(\mathrm{NCB})$ if they had any progressive disease or no reduction in tumour burden. This study was approved by the Institutional Review Board of DFCl.

A parallel cohort of $39 \mathrm{mUC}$ patients who received taxanebased chemotherapy, but did not receive ICls, was also analysed by similar methods.

\section{Data collection}

Clinical variables that were assessed included gender, race, age, smoking status, prior systemic chemotherapy regimens, prior radiotherapy, primary tumour site and site of lesion subjected to targeted sequencing. Baseline Eastern Cooperative Oncology Group (ECOG) performance status (PS), liver/visceral metastasis, haemoglobin $(\mathrm{Hb})$, NLR and platelet (PLT) count were captured at the time of $\mathrm{ICl}$ initiation (Table 1, S1.0).

Tissue collection and DNA extraction

Core biopsy and/or surgical resection specimens were reviewed by a BWH genitourinary pathologist (SS) to confirm the diagnosis, histological subtype, tumour grade and stage. Tumour regions consisting of at least $20 \%$ tumour cells were macrodissected from unstained slides, and DNA was isolated using the QIAamp DNA FFPE Tissue Kit (Qiagen) according to the manufacturer's instructions. DNA quantification was performed by Nanodrop and Pico-Green assays.

Tumour-targeted gene sequencing

Targeted gene sequencing was performed using an institutional analytic platform, Oncopanel, that is certified for clinical use and patients reporting under the Clinical Laboratory Improvement Amendments (CLIA) act. Genomic DNA from each tumour sample was subjected to targeted exon capture and sequencing using one of three versions of the Oncopanel assay (V1-V3) in the Department of Pathology at Brigham and Women's Hospital (BWH). The Oncopanel gene panel includes capture probes for 275-447 cancer-associated genes, as well as intronic portions of 60 genes for rearrangement detection. ${ }^{24}$ We focused our mutational and copy-number variation (CNV) analysis on 237 genes that were common to all versions of Oncopanel (Table S1.1).
Table 1. Clinical characteristics of the $\mathrm{mUC}$ patients who received $\mathrm{ICl}$, and were assessed for response.

\begin{tabular}{|c|c|}
\hline & Number (\%), $[n=62]$ \\
\hline \multicolumn{2}{|c|}{ Clinical/demographic variables } \\
\hline \multicolumn{2}{|l|}{ Age, years } \\
\hline Mean, range & $65.6,41.0-84.0$ \\
\hline \multicolumn{2}{|l|}{ Gender } \\
\hline Male & $45(73)$ \\
\hline Female & $17(27)$ \\
\hline \multicolumn{2}{|l|}{ ECOG PS } \\
\hline 0 & $26(44)$ \\
\hline 1 & $25(42)$ \\
\hline$\geq 2$ & $8(14)$ \\
\hline Missing & 3 \\
\hline \multicolumn{2}{|l|}{ Site } \\
\hline Bladder & $46(74)$ \\
\hline Upper tract & $16(26)$ \\
\hline \multicolumn{2}{|c|}{ Radical cystectomy/nephrouretrectomy } \\
\hline Yes & $43(69)$ \\
\hline No & $19(31)$ \\
\hline \multicolumn{2}{|c|}{ Previous lines of systemic therapy } \\
\hline 0 & $15(24)$ \\
\hline 1 & $36(58)$ \\
\hline$\geq 2$ & $11(18)$ \\
\hline \multicolumn{2}{|c|}{ Visceral metastases (bone, lungs, liver, etc.) } \\
\hline Yes & $45(73)$ \\
\hline No & $17(27)$ \\
\hline \multicolumn{2}{|c|}{ Neutrophil/lymphocyte ratio } \\
\hline Mean, range & $5.0,0.5-18.3$ \\
\hline \multicolumn{2}{|l|}{ Haemoglobin (g/dl) } \\
\hline Mean, range & $12.0,8.0-16.5$ \\
\hline \multicolumn{2}{|l|}{ Platelet count } \\
\hline Mean, range & $240,60-588$ \\
\hline \multicolumn{2}{|l|}{ Genomic factors } \\
\hline \multicolumn{2}{|l|}{ SNV count } \\
\hline Mean, range & $9.7,1.0-32.0$ \\
\hline \multicolumn{2}{|l|}{ CNV count } \\
\hline 0 & $29(47)$ \\
\hline 1 & $19(31)$ \\
\hline$\geq 2$ & $14(23)$ \\
\hline \multicolumn{2}{|c|}{$\mathrm{C}>\mathrm{T} \_\mathrm{CpG}$ mutation signature count } \\
\hline Mean, range & $2.6,0.0-9.5$ \\
\hline \multicolumn{2}{|c|}{ ERCC2 mutation signature count } \\
\hline Mean, range & $2.2,0.0-7.8$ \\
\hline \multicolumn{2}{|c|}{ APOBEC mutation signature count } \\
\hline Mean, range & $4.0,0.0-19.5$ \\
\hline \multicolumn{2}{|l|}{$C D K N 2 B$} \\
\hline Homozygous deletion & $14(23)$ \\
\hline No & $48(77)$ \\
\hline \multicolumn{2}{|c|}{ DNA damage repair (DDR) gene alteration (inclusive approach) } \\
\hline Yes & $34(55)$ \\
\hline No & $28(45)$ \\
\hline
\end{tabular}

The mean depth of read coverage for the targeted genes was $294 \times$ (Table S1.0). The single-nucleotide variant (SNV) count was defined as the number of exonic non-synonymous mutations per sample, including indels, nonsense mutations, splice site mutations and non-synonymous missense variants. In other reports, this is often referred to as tumour mutation burden (TMB). 
Variant assessment

We did not have sequencing information for germline DNA as part of this study. We excluded tumour sequence sequencing variants that were observed at a frequency $>0.1 \%$ in the Exome Aggregation Consortium (ExAC) database, ${ }^{25}$ as they were considered likely germline variants. All loss-of-function variants were considered deleterious, including nonsense mutations, frameshift indels or splice site alterations affecting consensus nucleotides. The functional impact of missense mutations was determined using SIFT $^{26}$ and Polyphen-2. ${ }^{27}$ Missense mutations classified as "damaging" in SIFT and/or "probably damaging" in Polyphen-2 were deemed deleterious (Table S1.3). Oncopanel covers 30 DNA damage repair (DDR) genes previously described in the literature (Table S1.4). ${ }^{28}$ Special consideration was given to missense variant assessment for DDR genes since prior studies have reported an association with benefit in the setting of chemotherapy or ICls. ${ }^{19,29-31}$ For DDR genes, we used two different strategies to define significant mutations. The first, a more inclusive method, included all loss-of-function variants as well as missense variants judged significant by either Polyphen- $2^{27}$ or SIFT, ${ }^{26}$ as described above (Table S1.5). The second, a more restrictive definition, included all loss-of-function alterations, and missense variants that met any of the following criteria: seen at least five times in the Catalogue of Somatic Mutations in Cancer (COSMIC) database, ${ }^{32}$ reported in OncoKB ${ }^{33}$ or reported in cancerhotspots.org ${ }^{34}$ (Table S1.6). For $E R C C 2$, all missense alterations within or near conserved helicase domains ${ }^{35}$ were included.

\section{Mutation signature analysis}

SNVs in the 62 samples were classified into 96 base substitution types within the trinucleotide sequence context that includes the bases immediately $5^{\prime}$ and $3^{\prime}$ to each altered base. Mutation signature analysis was performed to resolve the SNVs for each sample into a set of characteristic patterns (signatures) to infer the contributions of each signature in each tumour. ${ }^{36}$ The SNVs for each sample were projected onto the four mutation signatures (APOBEC-a, APOBEC-b, ERCC2 and C>T transitions at CpG dinucleotides) known to occur commonly in bladder carcinoma. ${ }^{37}$ This yielded a count of the estimated number of mutations in each sample generated by each of the four mutational processes (Table S1.7).

\section{Copy-number variant (CNV) analysis}

CNVs were identified using a custom R-based tool (VisCapCancer $^{28}$ that compares read depth at all genomic regions assayed among different samples. We focused on the most reliable CNVs in this analysis, homozygous deletions and amplifications, the latter defined as $>6$ copies. ${ }^{38,39}$ We calculated the CNV count as the total number of these two CNV events for each sample, considering only homozygous deletions in tumour-suppressor genes and amplifications in proto-oncogenes (Table S1.8).

\section{Statistical analysis}

Statistical tests included the Chi-Square and Fisher's exact tests for categorical variables and the Mann-Whitney $U$ test (two-group comparisons) or the Kruskal-Wallis test (three-group comparisons) for continuous variables. Associations between nine clinical and seven genomic features and clinical outcomes were assessed using univariable and multivariable binary logistic and Cox regression model. The primary clinical outcome was to examine the associations with clinical benefit. Secondary outcomes included overall survival (OS), and progression-free survival (PFS). OS was calculated from the start date of $\mathrm{ICl}$ therapy to the date of death or the last follow-up. Patients alive were censored at the date of the last contact. Progression-free survival (PFS) was calculated from the start date of $\mathrm{ICl}$ therapy to the date of progression, death or the last follow-up. Patients alive and progression-free were censored at the date of the last disease assessment.
Multivariable binary logistic (for NCB vs. CB) and Cox (for PFS and $O S$ ) regression models were fitted to the data using the variables selected by the ALASSO method. ${ }^{40}$ Regression coefficients were estimated in univariable analysis, separately for clinical and genomic variables, using binary logistic (for NCB vs. CB) and Cox proportional hazards (for PFS and OS) regression models, for each variable selected by the ALASSO method. Using a two-sided $p \leq 0.05$ criterion, variables from univariable analyses were selected for inclusion in the multivariable model with a stay criterion of $\leq 0.10$; the same stay criterion was used for the combined model containing clinical and genomic variables. Model discrimination performance was assessed using the area under the ROC curve (AUC), referred to as c-statistic (or c-index) for PFS and OS. See supplementary material 1 for additional details on model building and statistical analysis.

\section{RESULTS}

Patient and treatment characteristics

From June 2013 through December 2017, 102 mUC patients received PD-1/PD-L1 inhibitors at our institution and 67 of these patients had targeted next-generation tumour sequencing (Oncopanel) analysis performed on a primary or metastatic specimen. Of these, 62 patients were considered evaluable for response and were included in the analysis (Table 1, Fig. S1). The median patient age was 67 years (range, 41-84 years) and the majority of patients were male $(73 \%)$. Fifteen patients $(24 \%)$ were treated with $\mathrm{ICl}$ as first-line treatment for metastatic disease, while the remaining patients received chemotherapy prior to an $\mathrm{ICl}$. Most patients received the anti-PD-L1 agent atezolizumab (61\%) or the anti-PD-1 agent, pembrolizumab (31\%; Table S1.0). The majority of samples that were analysed by sequencing were primary tumours $(46 / 62$, $74 \%)$, and were obtained prior to the patient receiving an ICI (57/ $62,92 \%$; Table S2). Twenty-four (39\%) patients had CB in response to ICls, while $38(61 \%)$ did not. A separate cohort of 39 patients who received a taxane were identified (Table S2.0, Table S2.1). Seventeen (44\%) patients received docetaxel-based regimens, while the remaining $56 \%$ were treated with paclitaxel-based regimens. Sixteen patients received sequential taxane and $\mathrm{ICl}$ therapy, and 11 of these 16 had discordant clinical benefit between ICls and taxanes.

SNV count/TMB is associated with clinical benefit to ICI The association between single-nucleotide variant (SNV) count, determined by Oncopanel analysis (see the "Methods" section), and $\mathrm{ICl}$ response was examined. A median of 8 SNVs were identified in the 62 tumours analysed (range 1-32 SNVs). Patients with $C B$ had a significantly higher SNV count than patients with NCB (CB: median 13, range 4-32 vs. NCB: median 7, range, 1-15, $p<0.001$, Fig. 1a). Higher SNV count was also associated with longer progression-free survival (median PFS 6.01 vs. 1.97 months for patients with $\geq$ median SNV count vs. those with $<$ median SNV count, respectively, $p=0.002$ ).

APOBEC mutagenic signature is also associated with $C B$ to $\mathrm{ICI}$ The mutation signature, or pattern of specific mutations seen, in a tumour is a reflection of the different mutational processes active during its development. In the TCGA muscle-invasive bladder cancer cohort, ${ }^{37}$ two mutational signatures associated with apparent aberrant activity of the APOBEC cytosine deaminase accounted for $67 \%$ of all SNVs and were strongly associated with TMB. ${ }^{37}$ To investigate the relationship between mutational signature activity and $\mathrm{ICl}$ response, we projected all mutations in our cohort onto the three most common mutation signatures (fusing together two closely related APOBEC signatures) defined in the TCGA data. The number of estimated C>T_CpG mutations and estimated APOBEC-related mutations were both significantly higher in mUC patients with $C B$ to $\mathrm{ICl}$, whereas the number of 

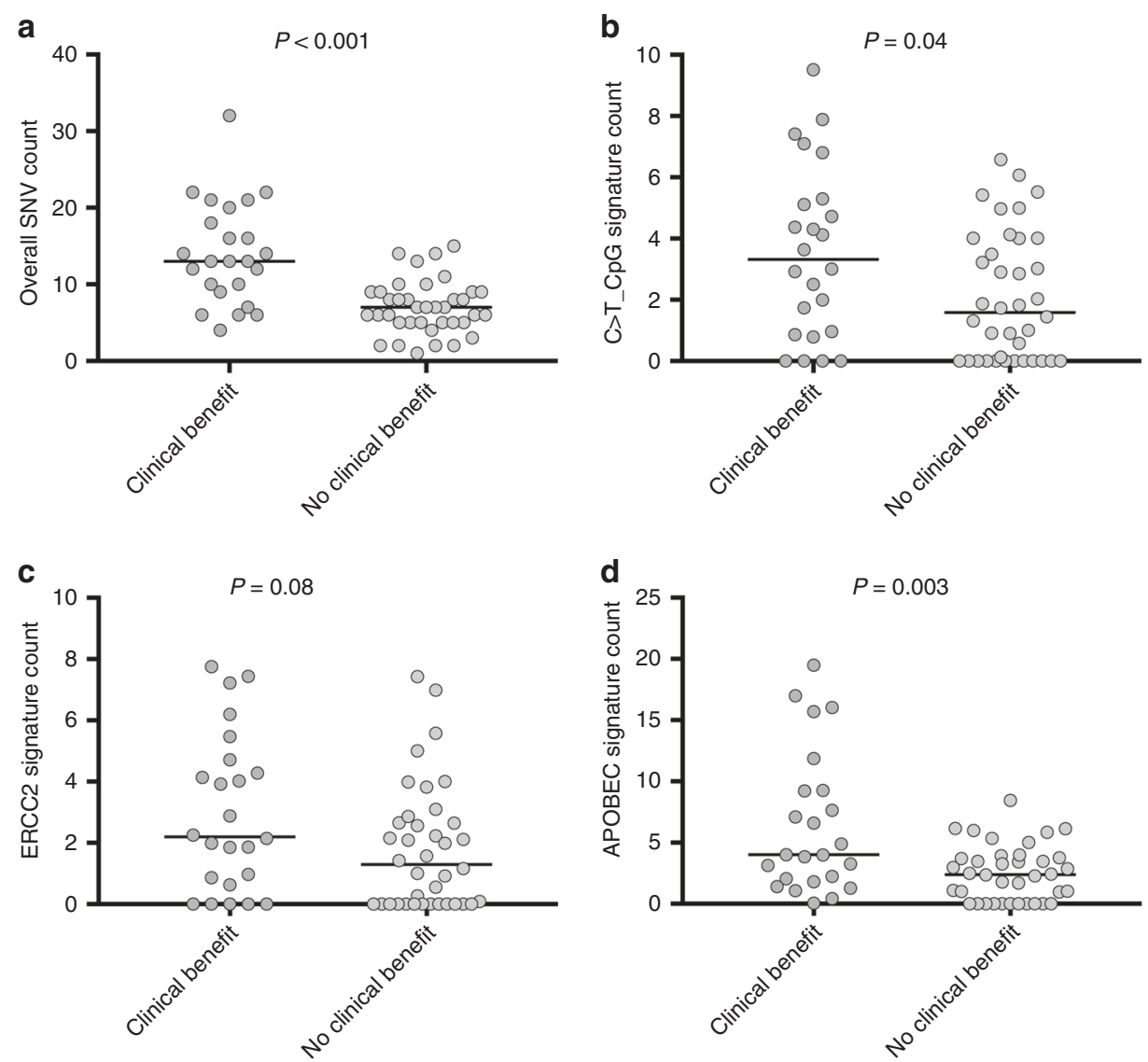

Fig. 1 Association between SNV count and clinical benefit to ICI in mUC. Dot plots are shown for a overall SNV count, b C>T@CpG signature mutation count, $\mathbf{c}$ ERCC2 signature mutation count and $\mathbf{d}$ APOBEC signature mutation count. Each dot represents a patient.

estimated ERCC2 mutations showed a trend to be higher in mUC with $\mathrm{CB}$ to $\mathrm{ICl}(p=0.041,0.003$ and 0.078 , respectively, Mann-Whitney $U$ test, Fig. $1 \mathrm{~b}-\mathrm{d}$ ). However, none of the three mutational signatures were associated with PFS or OS (Table 2).

CNV count and homozygous CDKN2B deletions are associated with NCB to ICI

Since aneuploidy is associated with reduced immune-mediated cytotoxic function, ${ }^{41,42}$ and CNV counts are associated with reduced survival in other cancer types, ${ }^{43,44}$ we examined the association between CNV count (see "Methods" section) and clinical outcomes in this cohort. The median CNV count was one (range 0-4). Higher CNV counts were seen in patients with NCB compared with patients with $\mathrm{CB}(p<0.001$, Mann-Whitney $U$ test, Fig. S2A). Although there is a potential confounding effect of tumour purity on our measurement of CNV, we did not find an association between tumour purity and CB (Fig. S2D). Nonetheless, there was a significant association between CNV count and tumour purity ( $p=0.012$, Fig. S2B), but not SNV count (Fig. S2C), consistent with an effect of tumour purity on the sensitivity of detection of CNV events. Higher CNV count was also associated with poorer PFS (hazard ratio [HR], 1.42; 95\% confidence interval [CI], 1.11-1.81; $p=0.01$, Table 2), but not OS.

Homozygous deletion of $C D K N 2 A$ and $C D K N 2 B$ was the most common CNV event in this cohort. CDKN2A and CDKN2B are located in close proximity (within $50 \mathrm{~kb}$ ) on chromosome $9 \mathrm{p}$, and their loss was concordant in the majority of samples (Fig. 2, Table S1.8). Homozygous deletions in CDKN2A and CDKN2B were each strongly associated with NCB in this cohort, with a slightly stronger effect for $C D K N 2 B$ homozygous deletions (OR for $N C B=$ $0.08 ; p=0.02$, Table 2). In addition, CDKN2B homozygous deletion was significantly associated with both worse PFS (HR=3.84; $p<$ $0.001)$ and $\mathrm{OS}(\mathrm{HR}=4.12 ; p=0.003)$.

Interaction between SNV and CNV counts and response to $\mathrm{ICl}$ Next, we considered SNV and CNV counts in combination and determined their association with response. Patients were classified into four subsets based on SNV (high or low) and CNV (high or low) counts, using the median of each count to divide the groups (Fig. S3). In total, 13 of 17 (76\%) patients with a combination of high SNV $(\geq 8)$ and low $\operatorname{CNV}(0)$ had $C B$, while none of 15 with low SNV $(<8)$ and high CNV $(>0)$ counts had CB. Patients with high SNV and CNV, or low SNV and CNV had intermediate response rates, 6 of 18 (33\%) and 5 of $12(42 \%)$, respectively ( $p<0.001$, Chi-square test). Despite the small sample sizes, these combined SNV-CNV subsets demonstrated a highly significant difference in PFS ( $p<0.0001$, Fig. 3).

DDR genes and response to $\mathrm{ICl}$ therapy

DDR gene alterations have been reported to be associated with response to ICls in mUC. ${ }^{19}$ In our cohort, we used two approaches to define significant mutations in DDR genes, one more inclusive and the other more restrictive (see "Methods" section). Using the more inclusive criteria, DDR gene alterations were identified in 34 of 62 (55\%) patients (Table S1.5). Tumours harbouring one or more DDR gene alterations had a significantly higher SNV count than tumours without DDR gene alterations ( $p=0.013$; Mann-Whitney $U$ test; Fig. S4). Given this correlation with SNV count, we studied the association between DDR gene alterations and clinical benefit using both the inclusive and restrictive criteria. Using both methods, we observed a trend towards enrichment of DDR gene alterations in the CB group, although statistical 
Table 2. Univariable analysis of clinical and molecular factors associated with CB vs. NCB, PFS and OS.

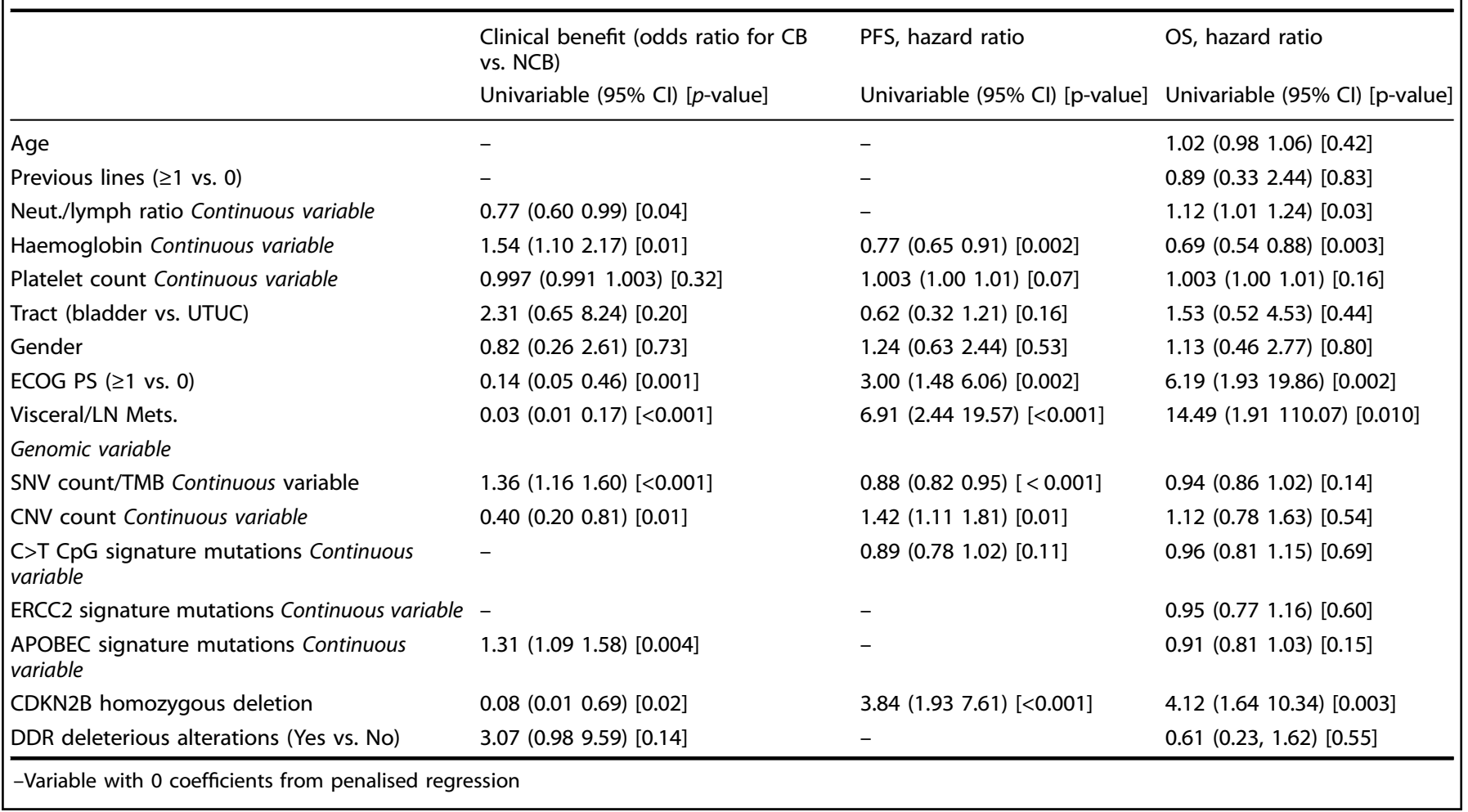

significance was not attained ( $p=0.192, p=0.129$, respectively, Fisher's exact test).

When individual DDR pathways were considered separately, both homologous recombination (HR) and nucleotide excision repair (NER) pathways demonstrated enrichment for mutations (using the more inclusive approach) in the $C B$ versus NCB groups $(7 / 24,29 \%$ vs. $1 / 38,3 \%, p=0.004 ; 5 / 24,21 \%$ vs. $1 / 38$, $3 \%, p=0.029$, respectively). Mutations in several individual genes were also enriched in the CB group: ERBB3 $(6 / 24,25 \%$ vs. $2 / 38,5.3 \%, p=0.047)$, MSH6 $(4 / 24,17 \%$ vs. $0 / 38,0 \%, p=0.019)$ and $B R C A 1(3 / 24,5 \%$ vs. $0 / 38,0 \%, p=0.054)$. However, because of the large number of genes evaluated for this association, none of these were significant after correction for multiple testing.

Association of clinical factors with $\mathrm{ICl}$ response

The presence of visceral metastases showed the strongest association with NCB (OR for NCB $=0.03, p<0.001$, Table 2) among all clinical variables considered. High NLR, low haemoglobin and low ECOG PS $(\geq 1)$ were also all associated with NCB $(\mathrm{OR}=$ $0.77, p=0.04 ; \mathrm{OR}=1.54, p=0.01 ; \mathrm{OR}=0.14, p=0.001$, respectively; Table 2). NLR was associated with PFS, and haemoglobin, ECOG PS and visceral metastases were all strongly correlated with both PFS and OS (Table 2).

Models combining clinical and genomic factors for clinical benefit To develop a robust predictor of clinical benefit to ICl therapy, we combined the molecular, clinical and laboratory factors associated with benefit in a single analysis and performed a multivariable analysis using ALASSO (see "Methods" section) to define independent predictors of response. Lack of visceral metastases, NLR $<5$ and high SNV count $(\geq 10)$ were all significantly associated with CB versus NCB, with a C-statistic $(95 \% \mathrm{Cl})$ of $0.90(0.80,0.99)$ (Table 3, Fig. S5). These three factors along with the CNV count for each patient and the extent of response to $\mathrm{ICl}$ according to RECIST 1.1 criteria are shown in Fig. $3 a$.
A linear predictor of clinical benefit was calculated using the three independent predictors of response (visceral metastasis, NLR and SNV count) including the intercept term (Table S4.1). In this predictor, the baseline was +1 , visceral metastases scored as -2.5 , NLR $\geq 5$ as -2 and SNV count $\geq 10$ as +3 , giving a range of scores from -3.5 to 4 (Table 3, S4.1). A threshold of $\geq-1$ was determined to be optimal for prediction of CB. Excluding 7 patients due to lack of NLR data at the time of ICl initiation, 22 of 24 patients with CB had a point score $\geq-1$ (sensitivity $=92 \%$, Table S4.2), while 27 of 31 patients with NCB had point scores $<-1$ (specificity $=87 \%$, Table S4.2). Interestingly, no patient with visceral metastasis, NLR $\geq 5$ and SNV count $<10(n=12)$ had clinical benefit to ICI therapy (point score $=-3.5$, Table S4.1). In contrast, patients without visceral metastasis, NLR $<5$ and SNV $\geq 10(n=10)$ all derived clinical benefit to ICls (point score $=4$, Table S4.1).

Predictive value of the $\mathrm{ICI}$ response model

To assess whether the association between these three factors and response to $\mathrm{ICl}$ therapy was prognostic or predictive, we evaluated all 16 variables found to be prognostic on univariable analysis in the $\mathrm{ICl}$ cohort in a comparator group of 39 patients from our institution treated with taxanes (Table S2.5). None of these 16 variables were significantly associated with response to taxanes (Table S2.5).

Predictive model combining clinical and genomic factors for survival

Multivariable composite models for PFS and OS were also developed. Visceral metastasis, platelet count and both SNV and CNV counts were independent predictors of PFS (c-statistic $(95 \%$ $\mathrm{Cl})=0.77(0.75,0.79)$, Table S5.1). A prognostic index for PFS was calculated including these four components (see Supplemental Material 1 for details). Patients were divided into three risk groups based on their prognostic index score (low risk $<-0.29 ;-0.29 \leq$ intermediate risk <1.54; high risk $\geq 1.54$ ), and PFS varied significantly among risk groups (Fig. S6A). 

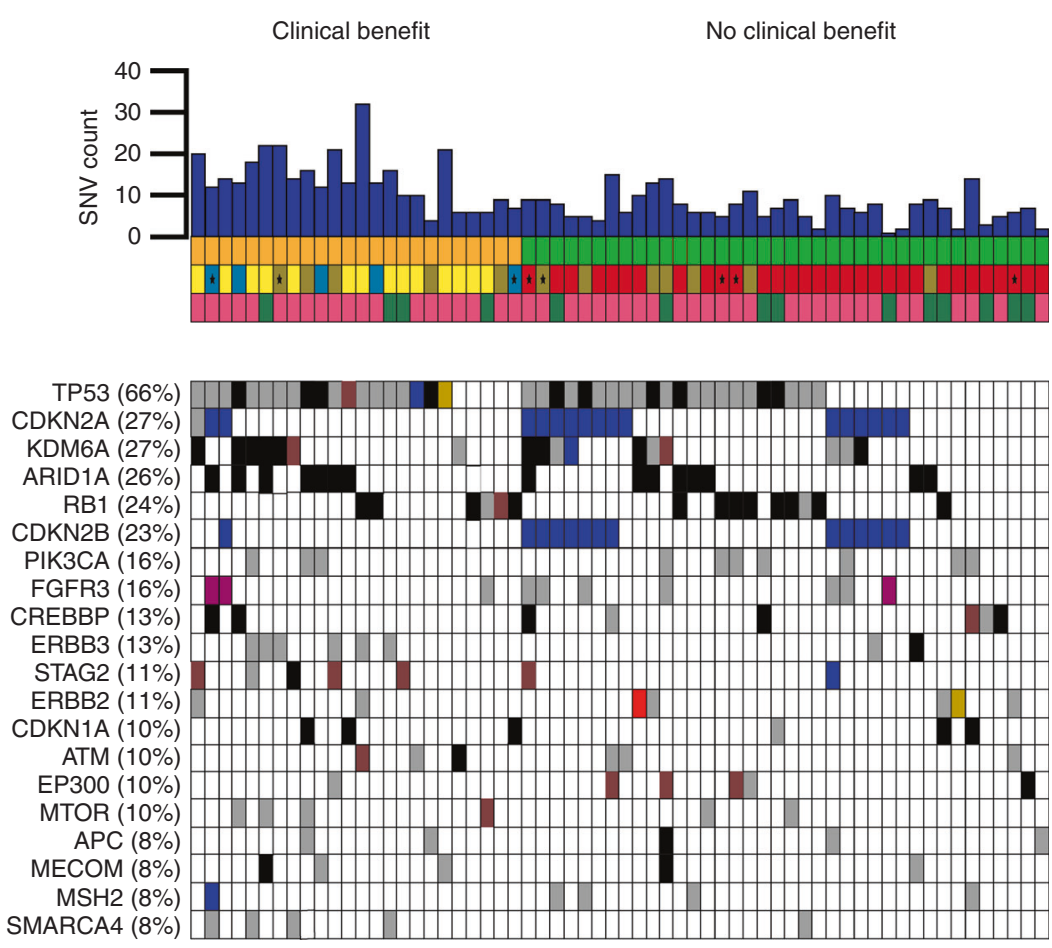

Clinical benefit

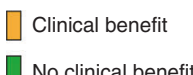

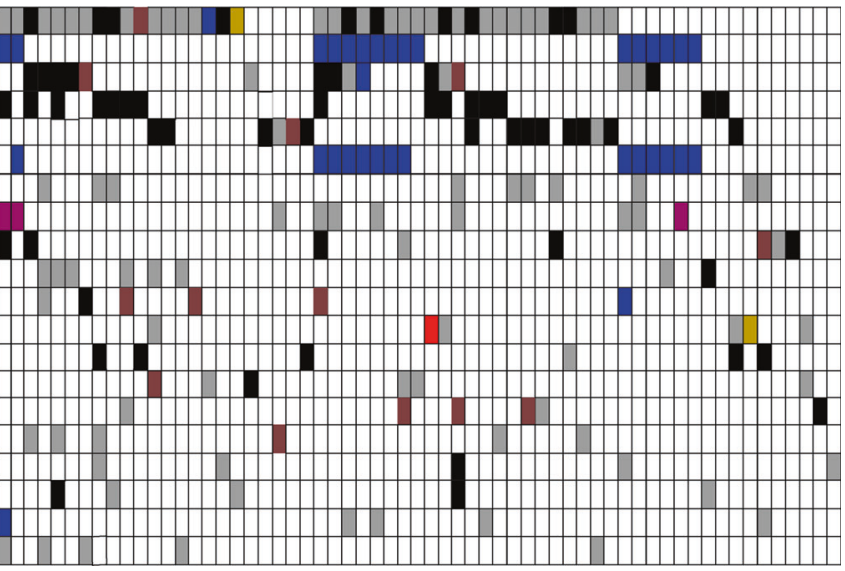

Site of urothelial carcinoma

Upper tract urothelial carcinoma

Bladder cancer

(1)
RECIST

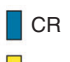

$\square \mathrm{PR}$

$\square \mathrm{SD}$

$\mathrm{PD}$

* Not evaluable by RECIST
Clinical benefit

RECIST

Site of urothelial carcinoma 
a

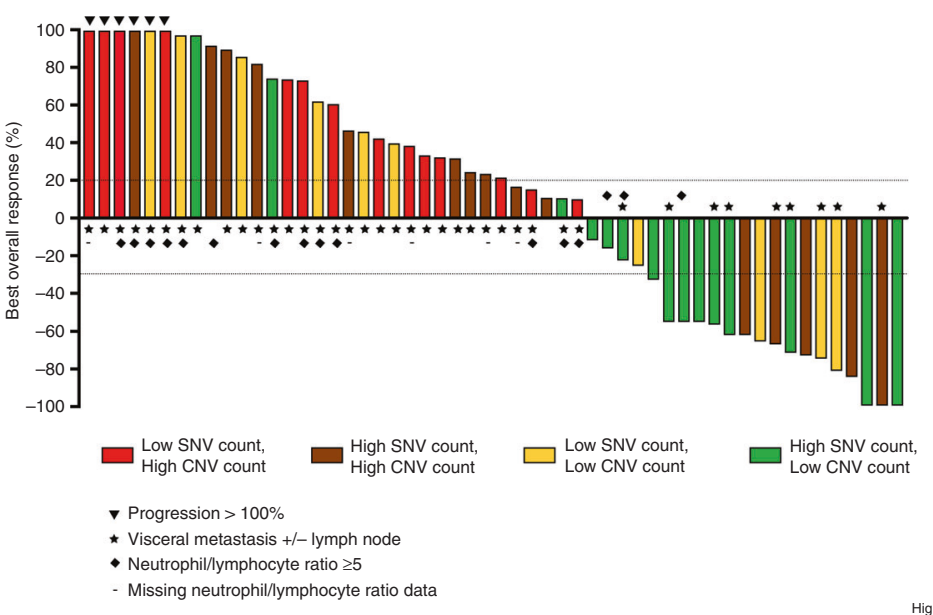

b

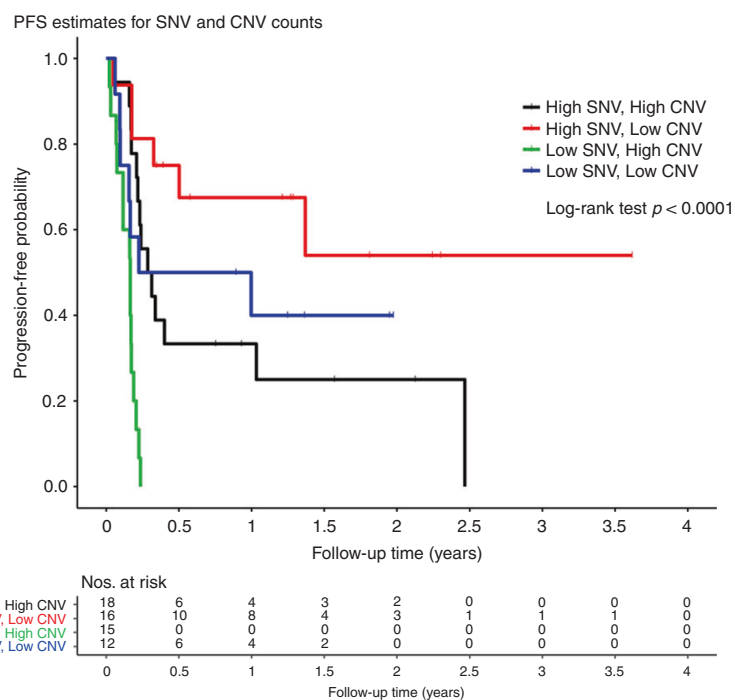

Fig. 3 Response to ICI according to SNV and CNV count, and clinical features. a Waterfall plot of response to ICI therapy, indicating SNV count, CNV count, the presence of visceral metastasis and NLR. b Progression-free survival (PFS) curve of UC patients in response to ICl, based on median cut-offs for SNVs (8) and CNVs (0 vs. $>0)$.

Table 3. Multivariable model for $\mathrm{CB}$ versus $\mathrm{NCB}$, containing clinical and genomic factors.

\begin{tabular}{lccrrr}
\hline Variable & \multicolumn{1}{c}{ Beta \pm SE } & OR $(95 \%$ Cl $)$ & $p$-value & Points & C-statistic $(95 \%$ Cl) \\
\hline Model intercept & $1.15 \pm 0.97$ & $3.16(0.47,21.14)$ & 0.237 & 1.0 & $0.90(0.80,0.99)$ \\
Visceral/LN Mets. & $-2.93 \pm 1.06$ & $0.05(0.01,0.43)$ & 0.006 & -2.5 \\
Neut/lymph ratio $(\geq 5$ vs. $<5)$ & $-2.11 \pm 1.14$ & $0.12(0.01,1.15)$ & 0.066 & -2.0 & 3.0 \\
SNV count $(\geq 10$ vs. $<10)$ & $3.21 \pm 0.97$ & $24.79(3.73,164.70)$ & $<0.001$ & \\
\hline
\end{tabular}

we used CNV count, including both homozygous deletions of tumour suppressors and high amplification of proto-oncogenes, as a quantitative measure of aneuploidy. In our analysis, both CNV count and CDKN2B homozygous deletions strongly associated with lack of response to $\mathrm{ICl}$ therapy. To our knowledge, this correlation with $C D K N 2 B$ deletions has not been reported in prior studies and may be intrinsic to $\mathrm{mUC}$ or be of broader relevance to other cancer types. CDKN2A and CDKN2B are located on chromosome 9p, and commonly deleted in bladder cancer, as well as other cancer types. $9 p$ deletions may extend to multiple other genes on $9 p$, including IFN and related pathway genes, so that this association may reflect perturbation in IFN signalling that contributes to immune exhaustion and lack of benefit from ICl therapy.

This retrospective analysis has several limitations. First, the relatively small number of patients studied limits the statistical power of our analysis. Second, we included patients who received $\mathrm{ICl}$ in the first-line as well as second-line setting; however, we assessed whether this difference contributed to differences in response, and it was not statistically significant. Third, patients were treated with different $\mathrm{ICl}$ regimens that may have variable efficacy. Fourth, most of the tumours subject to mutation analysis were primary lesions, and this eliminates the ability to detect clinically actionable alterations found exclusively in metastatic specimens. Nonetheless, this reflects common clinical practice. Fifth, Oncopanel does not cover all genes known to be mutated at significant frequency in $\mathrm{UC}^{37}$ including several genes that are commonly subject to amplification (E2F3, SOX4 and PPARG). Sixth, we were unable to determine with certainty whether variants were somatic versus germline and could not prove the functional effect of the missense variants identified. Finally, we did not have tumours from patients to perform PD-L1 immunohistochemistry
(IHC); however, given that IHC for PD-L1 has not been consistent as a prognostic factor for response to PD-1/PD-L1 inhibitors, ${ }^{52,53}$ we chose to focus on tumour genomics as a more robust and reproducible analysis that can have many clinical implications for management of mUC. High TMB has been reported to be associated with response to atezolizumab post platinum. ${ }^{54}$ Nonetheless, our findings are hypothesis-generating, and should be tested in larger and more uniformly treated cohorts.

\section{CONCLUSION}

In this cohort of $62 \mathrm{mUC}$ patients treated with ICl therapy, both a high and a low CNV count are strongly associated with response. Multivariable analysis identified lack of visceral metastasis, low NLR and high SNV as being independently predictive of clinical benefit to $\mathrm{ICl}$ but not to taxane-based chemotherapy. We used these features to generate a prediction score, which was highly correlated with clinical benefit [AUC $(95 \% \mathrm{Cl})=0.90(0.80,0.99)]$. These results highlight the power of combining readily available clinical and laboratory data with panel DNA sequencing data to stratify response to $\mathrm{ICl}$ in mUC. External validation of this predictive model in other cohorts is warranted.

\section{ACKNOWLEDGEMENTS}

None.

\section{AUTHOR CONTRIBUTIONS}

A.H.N.: conceptualisation, data curation, formal analysis, investigation, methodology, project administration, resources, software, supervision, validation, visualisation, 
writing-original draft and writing-review and editing. K.W.M.: conceptualisation, formal analysis, investigation, methodology, project administration, resources, software, supervision, validation, visualisation, writing —original draft and writingreview and editing. O.J.: formal analysis, software, validation, review and editing. A.B. S.: data curation, methodology, review and editing. J.K.: formal analysis, software, validation, review and editing. C.J.L.: formal analysis, software, validation, review and editing. M.P.: project administration, validation, review and editing. L.C.H.: project administration, validation, review and editing. E.M.V.A.: project administration, validation, review and editing. X.X.W.: project administration, validation, review and editing. B.M.: project administration, validation, review and editing. A.D.C.: project administration, validation, review and editing. M.P.: project administration, validation, review and editing. F.D.: project administration, validation, review and editing. S.S.: project administration, validation, review and editing. N.I.L.: project administration, validation, review and editing. J.B.: project administration, validation, review and editing. T.K.C.: conceptualisation, formal analysis, investigation, methodology, project administration, resources, software, supervision, validation, visualisation, writingoriginal draft and writing-review and editing. G.S.: conceptualisation, formal analysis, investigation, methodology, project administration, resources, software, supervision, validation, visualisation, writing—original draft and writing-review and editing. D.J.K.: conceptualisation, formal analysis, investigation, methodology, project administration, resources, software, supervision, validation, visualisation, writingoriginal draft and writing-review and editing.

\section{ADDITIONAL INFORMATION}

Ethics approval and consent to participate The study was initiated after approval from the GELB committee. The Dana-Farber Cancer Institute Institutional review boards (IRB) approval was obtained under protocol DF/HCC 11-104 and was conducted in accordance with the Declaration of Helsinki. A Dana-Farber Cancer Institute approved written informed consent was obtained from all patients.

\section{Consent to publish Not applicable.}

Data availability The datasets supporting the conclusions of this article are included within the article and its Supplementary material.

Competing interests Guru Sonpavde: consultant for BMS, Exelixis, Bayer, Sanofi, Pfizer, Novartis, Eisai, Janssen, Amgen, Astrazeneca, Merck, Genentech, EMD Serono and Astellas/Agensys; Research support to the institution from Astrazeneca, Bayer, Amgen, Boehringer-Ingelheim, Janssen, Merck, Sanofi and Pfizer; Author for Uptodate; Steering committee for Astrazeneca, BMS, Bavarian Nordic and Astellas; Speaker for Onclive; Research to Practice; Physician Education Resource (PER). Lauren C. Harshman reports consulting fees from Genentech, Dendreon, Pfizer, Medivation/ Astellas, Exelixis, Bayer, Kew Group, Corvus, Merck, Novartis, Michael J. Hennessy Associates (Healthcare Communications Company and several brands such as OncLive and PER), Jounce and EMD Serono; Research funding from Bayer, Sotio, Bristol-Myers Squib, Merck, Takeda, Dendreon/Valient, Jannsen, Medivation/Astellas, Genentech, Pfizer and Endocyte (Novartis), and support for research travel from Bayer and Genentech. David J. Kwiatkowski reports serving as a consultant for Novartis. Eliezer Van Allen reported serving as an advisor or consultant for Tango Therapeutics, Genome Medical, Invitae, Illumina, Foresite Capital and Dynamo. He receives research support from Novartis, BMS; has equity in Tango Therapeutics, Genome Medical, Syapse and Microsoft; receives travel reimbursement from Roche/Genentech; holds institutional patents on ERCC2 mutations and chemotherapy response, chromatin mutations and immunotherapy response, and methods for clinical interpretation. Toni K. Choueiri reported receiving institutional and personal funds from Analysis Group, AstraZeneca, Alexion, Bayer, Bristol-Myers-Squibb/ER Squibb and sons LLC, Cerulean, Eisai, Foundation Medicine Inc., Exelixis, Ipsen, Tracon, Genentech, Roche, Roche Products Limited, F. Hoffmann-La Roche, GlaxoSmithKline, Lilly, Merck, Novartis, Peloton, Pfizer, Prometheus Labs, Corvus, Calithera, Sanofi/Aventis and Takeda; reported receiving honoraria from the Analysis Group, AstraZeneca, Alexion, Sanofi/Aventis, Bayer, Bristol-Myers-Squibb/ER Squibb and sons LLC, Cerulean, Eisai, Foundation Medicine Inc., Exelixis, Genentech, Roche, Roche Products Limited, F. Hoffmann-La Roche, GlaxoSmithKline, Heron Therapeutics, Lilly, Merck, Novartis, Peloton, Pfizer, EMD Serono, Prometheus Labs, Corvus, Ipsen, Up-to-Date, NCCN, Analysis Group, NCCN, Michael J. Hennessy (MJH) Associates, Inc. (Healthcare Communications Company with several brands such as OnClive, PeerView and PER), L-path, Kidney Cancer Journal, Clinical Care Options, Platform Q, Navinata Healthcare, Harborside Press, American Society of Medical Oncology, NEJM and Lancet Oncology; having a consulting or advisory role at Analysis Group, AstraZeneca, Alexion, Sanofi/ Aventis, Bayer, Bristol-Myers-Squibb/ER Squibb and sons LLC, Cerulean, Eisai, Foundation Medicine Inc., Exelixis, Genentech, Heron Therapeutics, Lilly, Roche, GlaxoSmithKline, Merck, Novartis, Peloton, Pfizer, EMD Serono, Prometheus Labs, Corvus, Ipsen, Up-to-Date and NCCN. No speaker's bureau. No leadership or employment in for-profit companies. Other present or past leadership roles: director of GU Oncology Division at Dana-Farber and past president of medical staff at DanaFarber, member of NCCN Kidney panel and the GU Steering Committee and past chairman of the Kidney Cancer Association Medical and Scientific Steering Committee. Patents, royalties or other intellectual properties: International Patent Application No. PCT/US2018/12209, entitled "PBRM1 Biomarkers Predictive of AntiImmune Checkpoint Response," filed January 3, 2018, claiming priority to U.S. Provisional Patent Application No. 62/445,094, filed January 11, 2017; International Patent Application No. PCT/US2018/058430, entitled "Biomarkers of Clinical Response and Benefit to Immune Checkpoint Inhibitor Therapy," filed October 31, 2018, claiming priority to U.S. Provisional Patent Application No. 62/581,175, filed November 3, 2017. Travel, accommodations and expenses, in relation to consulting, advisory roles or honoraria. Medical writing and editorial assistance support may have been funded by Communications companies funded by pharmaceutical companies (ClinicalThinking, Envision Pharma Group, Fishawack Group of Companies, Health Interactions, Parexel and others). The institution (Dana-Farber Cancer Institute) may have received additional independent funding of drug companies or/ and royalties potentially involved in research around the subject matter. CV provided upon request for scope of clinical practice and research. Kent W. Mouw reports consulting fees from Pfizer, EMD Serono and OncLive, as well as research funding from Pfizer

Funding information Not applicable.

Supplementary information is available for this paper at https://doi.org/10.1038/ s41416-019-0686-0.

Note: This work is published under the standard license to publish agreement. After 12 months the work will become freely available and the license terms will switch to a Creative Commons Attribution 4.0 International (CC BY 4.0).

Publisher's note Springer Nature remains neutral with regard to jurisdictional claims in published maps and institutional affiliations.

\section{REFERENCES}

1. Hugo, W., Zaretsky, J. M., Sun, L., Song, C., Moreno, B. H., Hu-Lieskovan, S. et al. Genomic and transcriptomic features of response to anti-PD-1 Therapy in metastatic melanoma. Cell 165, 35-44 (2016).

2. Motzer, R. J., Escudier, B., McDermott, D. F., George, S., Hammers, H. J., Srinivas, S. et al. Nivolumab versus Everolimus in advanced renal-cell carcinoma. N. Engl. J. Med. 373, 1803-1813 (2015).

3. Seiwert, T. Y., Burtness, B., Mehra, R., Weiss, J., Berger, R., Eder, J. P. et al. Safety and clinical activity of pembrolizumab for treatment of recurrent or metastatic squamous cell carcinoma of the head and neck (KEYNOTE-012): an open-label, multicentre, phase 1b trial. Lancet Oncol. 17, 956-965 (2016).

4. Necchi, A., Joseph, R. W., Loriot, Y., Hoffman-Censits, J., Perez-Gracia, J. L., Petrylak, D. P. et al. Atezolizumab in platinum-treated locally advanced or metastatic urothelial carcinoma: post-progression outcomes from the phase II IMvigor210 study. Ann Oncol. 28, 3044-3050 (2017).

5. Rosenberg, J. E., Hoffman-Censits, J., Powles, T., van der Heijden, M. S., Balar, A. V., Necchi, A. et al. Atezolizumab in patients with locally advanced and metastatic urothelial carcinoma who have progressed following treatment with platinum-based chemotherapy: a single-arm, multicentre, phase 2 trial. Lancet 387, 1909-1920 (2016).

6. Massard, C., Gordon, M. S., Sharma, S., Rafii, S., Wainberg, Z. A., Luke, J. et al. Safety and efficacy of durvalumab (MEDI4736), an anti-programmed cell death ligand-1 immune checkpoint inhibitor, in patients with advanced urothelial bladder cancer. J. Clin. Oncol. 34, 3119-3125 (2016).

7. Bellmunt, J., Sonpavde, G., Wit, R. D., Choueiri, T. K., Siefker-Radtke, A. O., Plimack, E. R. et al. KEYNOTE-045: randomized phase 3 trial of pembrolizumab (MK-3475) versus paclitaxel, docetaxel, or vinflunine for previously treated metastatic urothelial cancer. J. Clin.Oncol. 33, TPS4571-TPS4571 (2015).

8. Cheng, W., Fu, D., Xu, F. \& Zhang, Z. Unwrapping the genomic characteristics of urothelial bladder cancer and successes with immune checkpoint blockade therapy. Oncogenesis 7, 2 (2018).

9. Le, D. T., Durham, J. N., Smith, K. N., Wang, H., Bartlett, B. R., Aulakh, L. K. et al. Mismatch repair deficiency predicts response of solid tumors to PD-1 blockade. Science 357, 409-413 (2017).

10. Taube, J. M., Klein, A., Brahmer, J. R., Xu, H., Pan, X., Kim, J. H. et al. Association of PD-1, PD-1 ligands, and other features of the tumor immune microenvironment with response to anti-PD-1 therapy. Clin. Cancer Res. 20, 5064-5074 (2014).

11. Gibney, G. T., Weiner, L. M. \& Atkins, M. B. Predictive biomarkers for checkpoint inhibitor-based immunotherapy. Lancet Oncol. 17, e542-e551 (2016). 
12. Asaoka, Y., ljichi, H. \& Koike, K. PD-1 Blockade in Tumors with Mismatch-Repair Deficiency. N. Engl. J. Med. 373, 1979 (2015).

13. Rizvi H., Sanchez-Vega F., La K., Chatila W., Jonsson P., Halpenny D., et al. Molecular determinants of response to anti-programmed cell death (PD)- 1 and antiprogrammed death-ligand (PD-L)-ligand 1 blockade in patients with non-smallcell lung cancer profiled with targeted next-generation sequencing. J. Clin. Oncol. 36, 633-641. (2018).

14. Lauss, M., Donia, M., Harbst, K., Andersen, R., Mitra, S., Rosengren, F. et al. Mutational and putative neoantigen load predict clinical benefit of adoptive $T$ cell therapy in melanoma. Nat. Commun. 8, 1738 (2017).

15. McGranahan, N., Furness, A. J., Rosenthal, R., Ramskov, S., Lyngaa, R., Saini, S. K. et al. Clonal neoantigens elicit $\mathrm{T}$ cell immunoreactivity and sensitivity to immune checkpoint blockade. Science 351, 1463-1469 (2016).

16. Gubin, M. M., Zhang, X., Schuster, H., Caron, E., Ward, J. P., Noguchi, T. et al. Checkpoint blockade cancer immunotherapy targets tumour-specific mutant antigens. Nature 515, 577-581 (2014).

17. Rizvi, N. A., Hellmann, M. D., Snyder, A., Kvistborg, P., Makarov, V., Havel, J. J. et al. Cancer immunology. Mutational landscape determines sensitivity to PD-1 blockade in non-small cell lung cancer. Science 348, 124-128 (2015).

18. Roh W., Chen P. L., Reuben A., Spencer C. N., Prieto P. A., Miller J. P., et al. Integrated molecular analysis of tumor biopsies on sequential CTLA-4 and PD-1 blockade reveals markers of response and resistance. Sci. Transl. Med. 9, pii: eaah3560 (2017)

19. Teo, M. Y., Seier, K., Ostrovnaya, I., Regazzi, A. M., Kania, B. E., Moran, M. M. et al. Alterations in DNA damage response and repair genes as potential marker of clinical benefit from PD-1/PD-L1 blockade in advanced urothelial cancers. J. Clin. Oncol. 36, 1685-1694 (2018).

20. Ferrucci, P. F., Ascierto, P. A., Pigozzo, J., Del Vecchio, M., Maio, M., Antonini Cappellini, G. C. et al. Baseline neutrophils and derived neutrophil-to-lymphocyte ratio: prognostic relevance in metastatic melanoma patients receiving ipilimumab. Ann. Oncol. 27, 732-738 (2016).

21. Pond, G. R., Niegisch, G., Rosenberg, J. E., Dreicer, R., Powles, T., Necchi, A. et al. New 6-factor prognostic model for patients (pts) with advanced urothelial carcinoma (UC) receiving post-platinum atezolizumab. J. Clin. Oncol. 36413 (2018).

22. Nassar A. H., Umeton R., Kim J., Lundgren K., Harshman L. C., Van Allen E. M., et al. Mutational analysis of 472 urothelial carcinoma across grades and anatomic sites. Clin. Cancer Res. 25, 2458-2470 (2018).

23. Wolchok, J. D., Hoos, A., O'Day, S., Weber, J. S., Hamid, O., Lebbe, C. et al. Guidelines for the evaluation of immune therapy activity in solid tumors: immune-related response criteria. Clin. Cancer Res. 15, 7412-7420 (2009).

24. Sholl, L. M., Do, K., Shivdasani, P., Cerami, E., Dubuc, A. M., Kuo, F. C. et al. Institutional implementation of clinical tumor profiling on an unselected cancer population. JCI Insight. 1, e87062 (2016).

25. Lek, M., Karczewski, K. J., Minikel, E. V., Samocha, K. E., Banks, E., Fennell, T. et al. Analysis of protein-coding genetic variation in 60,706 humans. Nature 536, 285-291 (2016).

26. Kumar, P., Henikoff, S. \& Ng, P. C. Predicting the effects of coding nonsynonymous variants on protein function using the SIFT algorithm. Nat. Protoc. 4 1073-1081 (2009).

27. Adzhubei, I. A., Schmidt, S., Peshkin, L., Ramensky, V. E., Gerasimova, A., Bork, P. et al. A method and server for predicting damaging missense mutations. Nat. Methods. 7, 248-249 (2010).

28. Milanowska, K., Krwawicz, J., Papaj, G., Kosinski, J., Poleszak, K., Lesiak, J. et al. REPAIRtoire-a database of DNA repair pathways. Nucleic Acids Res. 39 D788-D792 (2011).

29. Teo, M. Y., Bambury, R. M., Zabor, E. C., Jordan, E., Al-Ahmadie, H., Boyd, M. E. et al. DNA damage response and repair gene alterations are associated with improved survival in patients with platinum-treated advanced urothelial carcinoma. Clin. Cancer Res. 23, 3610-3618 (2017).

30. Mariathasan, S., Turley, S. J., Nickles, D., Castiglioni, A., Yuen, K., Wang, Y. et al. TGFbeta attenuates tumour response to PD-L1 blockade by contributing to exclusion of T cells. Nature 554, 544-548 (2018).

31. Pietzak, E. J., Bagrodia, A., Cha, E. K., Drill, E. N., lyer, G., Isharwal, S. et al. Nextgeneration sequencing of nonmuscle invasive bladder cancer reveals potential biomarkers and rational therapeutic targets. Eur. Urol. 72, 952-959 (2017).

32. Forbes, S. A., Beare, D., Boutselakis, H., Bamford, S., Bindal, N., Tate, J. et al. COSMIC: somatic cancer genetics at high-resolution. Nucleic Acids Res. 45 D777-D783 (2017)
33. Chakravarty D., Gao J., Phillips S. M., Kundra R., Zhang H., Wang J., et al. OncoKB: a precision oncology knowledge base. JCO Precis Oncol. https://ascopubs.org/doi/ 10.1200/PO.17.00011 (2017).

34. Chang, M. T., Bhattarai, T. S., Schram, A. M., Bielski, C. M., Donoghue, M. T. A. Jonsson, P. et al. Accelerating discovery of functional mutant alleles in cancer. Cancer Discov. 8, 174-183 (2018).

35. Li Q., Damish A., Frazier Z. J., Liu D., Reznichenko E., Kamburov A., et al. ERCC2 helicase domain mutations confer nucleotide excision repair deficiency and drive cisplatin sensitivity in muscle-invasive bladder cancer. Clin. Cancer Res. 25 977-988 (2018).

36. Alexandrov, L. B., Nik-Zainal, S., Wedge, D. C., Aparicio, S. A., Behjati, S., Biankin, A. V. et al. Signatures of mutational processes in human cancer. Nature 500, 415-421 (2013).

37. Robertson, A. G., Kim, J., Al-Ahmadie, H., Bellmunt, J., Guo, G., Cherniack, A. D. et al. Comprehensive molecular characterization of muscle-invasive bladder cancer. Cell 171, 540-56 e25 (2017).

38. Pugh, T. J., Amr, S. S., Bowser, M. J., Gowrisankar, S., Hynes, E., Mahanta, L. M. et al. VisCap: inference and visualization of germ-line copy-number variants from targeted clinical sequencing data. Genet Med. 18, 712-719 (2016).

39. Garcia, E. P., Minkovsky, A., Jia, Y., Ducar, M. D., Shivdasani, P., Gong, X. et al. Validation of OncoPanel: a targeted next-generation sequencing assay for the detection of somatic variants in cancer. Arch. Pathol. Lab. Med. 141, 751-758 (2017).

40. Zou, H. The adaptive lasso and its oracle properties. J. Am. Stat.Assoc. 101 1418-1429 (2006).

41. Davoli T., Uno H., Wooten E. C. \& Elledge S. J. Tumor aneuploidy correlates with markers of immune evasion and with reduced response to immunotherapy. Science 355, pii: eaaf8399 (2017).

42. Ock, C. Y., Hwang, J. E., Keam, B., Kim, S. B., Shim, J. J., Jang, H. J. et al. Genomic landscape associated with potential response to anti-CTLA-4 treatment in cancers. Nat Commun. 8, 1050 (2017).

43. Kallioniemi, O. P., Punnonen, R., Mattila, J., Lehtinen, M. \& Koivula, T. Prognostic significance of DNA index, multiploidy, and S-phase fraction in ovarian cancer. Cancer 61, 334-339 (1988).

44. Clark, G. M., Dressler, L. G., Owens, M. A., Pounds, G., Oldaker, T. \& McGuire, W. L. Prediction of relapse or survival in patients with node-negative breast cancer by DNA flow cytometry. N Engl J Med. 320, 627-633 (1989).

45. Maleki Vareki, S. High and low mutational burden tumors versus immunologically hot and cold tumors and response to immune checkpoint inhibitors. J Immunother Cancer 6, 157 (2018).

46. Bowen, R. C., Little, N. A. B., Harmer, J. R., Ma, J., Mirabelli, L. G., Roller, K. D. et al. Neutrophil-to-lymphocyte ratio as prognostic indicator in gastrointestinal cancers: a systematic review and meta-analysis. Oncotarget 8, 32171-32189 (2017).

47. Hawinkels, L. J., Zuidwijk, K., Verspaget, H. W., de Jonge-Muller, E. S., van Duijn, W., Ferreira, V. et al. VEGF release by MMP-9 mediated heparan sulphate cleavage induces colorectal cancer angiogenesis. Eur J Cancer 44, 1904-1913 (2008).

48. Knaapen, A. M., Gungor, N., Schins, R. P., Borm, P. J. \& Van Schooten, F. J. Neutrophils and respiratory tract DNA damage and mutagenesis: a review. Mutagenesis 21, 225-236 (2006).

49. He, G., Zhang, H., Zhou, J., Wang, B., Chen, Y., Kong, Y. et al. Peritumoural neutrophils negatively regulate adaptive immunity via the PD-L1/PD-1 signalling pathway in hepatocellular carcinoma. J Exp Clin Cancer Res. 34, 141 (2015).

50. Pine, J. K., Morris, E., Hutchins, G. G., West, N. P., Jayne, D. G., Quirke, P. et al. Systemic neutrophil-to-lymphocyte ratio in colorectal cancer: the relationship to patient survival, tumour biology and local lymphocytic response to tumour. $\mathrm{Br} \mathrm{J}$ Cancer 113, 204-211 (2015).

51. Balkwill, F. \& Mantovani, A. Inflammation and cancer: back to Virchow? Lancet 357, 539-545 (2001).

52. Powles, T., Duran, I., van der Heijden, M. S., Loriot, Y., Vogelzang, N. J., De Giorgi, U. et al. Atezolizumab versus chemotherapy in patients with platinum-treated locally advanced or metastatic urothelial carcinoma (IMvigor211): a multicentre, open-label, phase 3 randomised controlled trial. Lancet 391, 748-757 (2018).

53. Bellmunt, J. \& Bajorin, D. F. Pembrolizumab for Advanced Urothelial Carcinoma. $N$ Engl J Med. 376, 2304 (2017).

54. Powles T., Loriot Y., Ravaud A., Vogelzang N. J., Duran I., Retz M., et al. Atezolizumab (atezo) vs. chemotherapy (chemo) in platinum-treated locally advanced or metastatic urothelial carcinoma (mUC): Immune biomarkers, tumor mutational burden (TMB), and clinical outcomes from the phase III IMvigor211 study. J. Clin. Onco. 36, 409 (2018). 